\title{
Localization of Laminin and Fibronectin in Rat Testes after Diisobutyl Phthalate Exposure: Histopathologic and Immunohistochemical Study
}

\author{
Yücel Başımoğlu Koca* \\ Aydın Adnan Menderes University, Faculty of Science and Arts, Department of Biology, Aydın, Turkey \\ *ybasimoglukoca@gmail.com
}

Received: 27 May 2019

Accepted: 17 September 2019

DOI: $10.18466 /$ cbayarfbe. 570613

\begin{abstract}
In this study the effects of Diisobutyl phthalate (DIBP) from commonly used pytalates were histopathologically investigated in order to find out whether it is one of the causes of increased infertility in recent years. In addition, the localization of laminin and fibronectin was demonstrated immunohistochemically after exposure to DIBP. For this purpose, three study groups from Wistar albino rats were created: experimental group, control group and corn oil applied group. The experimental group were administered by gavage 3 different doses (0.25-0.5-1 ml/kg/day) of DIBP mixed with corn oil every day for 28 days. At the end of the experiment, the testis tissue samples from all of the experimental and control group animals were investigated and evaluated with a light microscope after routine histological and immunohistochemical preparation processes. Reduction in spermatogenic cell population, apoptotic and necrotic germ cells, increase in Sertoli cells, multinucleate giant cells, thickening and dilatation in basal lamina of seminiferous tubules, oedema and shrinkage in interstitial connective tissue were observed in DIBP treatment groups. The decrease in localization of fibronectin and laminin immunoreactivity in seminiferous tubules was observed. The results of this study demonstrated that DIBP causes serious damage to the testicular tissue, especially in terms of spermatogenesis. For this reason, it has come to the conclusion that the material in question may cause infertility in male individuals.
\end{abstract}

Keywords: Diisobutyl phthalate, testes, laminin, fibronectin, histopathology.

\section{Introduction}

Phthalates are widely used as plasticizers in polyvinyl chloride (PVC) plastics. Diisobutyl phthalate (DIBP) $\left(\mathrm{C}_{16} \mathrm{H}_{22} \mathrm{O}_{4}\right)$ is a dialkyl phthalate ester phthalate which can be used as a substitute of dibutyl phthalate $[1,2]$. Many consumer products such as building materials, cosmetics, medical devices, food packaging, cleaning materials and children's toys contain phtalates. Humans are expose through ingestion, inhalation and dermal exposure during their whole lifetime, including intrauterine development [8]. It is known that some of the commonly used phthalates have adverse effects on the reproductive system of male rats after prenatal exposure [3, 4]. Some of these effects are reported to be caused by a decrease in testicular testosterone production in late pregnancy $[3,5,6]$.

In spermatogenesis, the connection and communication between cells is very important. The Extracellular Matrix (ECM) a specialized and dynamic structure plays an essential role in providing structural integrity. Its components (fibronectin, laminin, type IV collagen, vitronectin, tenascin, and pentactine) are ECM and filamentous glycoproteins that provide adhesion between cells $[7,8]$.
In this study the effects of Diisobutyl phthalate (DIBP) from commonly used phtalates were histopathologically investigated in order to determine whether it is one of the causes of increased infertility in recent years. In the functions such as cell-cell adhesion, signal transduction between cells, differentiation and cell migration to be carried out in a healthy manner fibronectin and laminin molecules are directly involved in the spermatogenesis. For this reason, in addition, in this study, the distribution in testes tissue of these two important molecules after DIBP exposure was immunohistochemically demonstrated.

\section{Material and Methods}

Diisobutyl phthalate (IBP; Cas No: 84-69-5) was purchased from Chemical Service Inc. (West Chester). All other chemicals were obtained from Sigma Chemical (St. Louis, MO). Firstly, approval by the Experimental Animals Local Ethics Committee, Adnan Menderes University (ADU) (confirmation number: 2013/006) was taken. Male Wistar albino rats (n: 20, mean $250 \mathrm{~g}$ weight) obtained from ADU-Faculty of Veterinary were used. Rats were kept at $22 \mathrm{C}^{0}$ with $14 \mathrm{~h}$ 
light, $10 \mathrm{~h}$ dark schedule and were fed standard food pellets and water ad libitum.

The study was divided into 5 different groups (total rat number: 25) (control group, corn oil group and DIBP group). Control group animals were not given any substance. Sham group animals were given only corn oil used as solvent. Exposure groups DIBP was administered orally at doses of $0.25 \mathrm{ml} / \mathrm{kg}$ (group 1), 0.5 $\mathrm{ml} / \mathrm{kg}$ (group 2), $1 \mathrm{ml} / \mathrm{kg}$ (group 3), $\mathrm{ml} / \mathrm{kg}$ after mixing with corn oil. After mixing with corn oil, DIBP was administered orally to experimental animals at doses of $0.25 \mathrm{ml} / \mathrm{kg}$ (group 1), $0.5 \mathrm{ml} / \mathrm{kg}$ (group 2), $1 \mathrm{ml} / \mathrm{kg}$ (group 3). The treatment dose of the DIBP was calculated based on LD50 (10.400 mg/kg body weight for mice) and body weight data. The animals of the treated groups were administered with DIBP with corn oil by oral route during 28 days as described in OECD guideline 407 [9].

Testicular tissue samples taken from all animals of control and experimental groups were processed to histological preparation. One testis of each rat was fixed in $10 \%$ neutral buffered formalin (NBF) solution, dehydrated and embedded in paraffin. Testis tissue blocks were sectioned on a Leica 2145 microtome at 5 $\mu \mathrm{m}$ thickness. Sections were stained with Hematoxylin\&Eosin and Periodic acid Schiff (PAS) [10]. The preparations obtained were evaluated in the light microscope (Olympus BX51) level.

In order to determine the laminin and fibronectin expression were applied a Peroksidaz-anti-peroksidaz (PAP) procedure. The testis tissues were sectioned at 7 $\mu \mathrm{m}$ thickness. And they were deparaffinized in the incubators at $60^{\circ} \mathrm{C}$, the pellucidity process with xylol was performed. Next, rehydration through the decreasing alcohol series was performed, and they were subsequently kept waiting in distilled water. The sections blocked with $3 \% \mathrm{H}_{2} \mathrm{O}_{2}$ to inhibit endogenous peroxides activity, and they were washed by means of phosphate buffer solution (PBS) and were treated with the blocking solution. Laminin (Vector Lab. Laminin VP L 551) and fibronectin (Vector Lab. Laminin VP F 705) primary antibodies were applied to sections in the humid chamber at room temperature for 1 hour. Immunoglobulin (Vector Lab. Vectastein Elit ABC Kit PK 6200) developed against the biotinylated mouse and rabbit as the secondary antibody was applied for 30 minutes in a humid environment at room temperature. The sections washed through PBS once again and then were stained with Diaminobenzidine tetrahydrochloride (DAB) (Vector Lab. Peroxide Sub. SK 4100) to identify the visibility of the immunohistochemical reaction. Counterstaining with hematoxylin was performed to show off the cell nuclei. After dehydration and stabilization with mounting medium, the sections were assessed by a light microscope. The intensity of brown color showed the level of laminin and fibronectin in sections. Image analysis was performed by quantitative scoring method (-; negative, +; very weak, ++; weak, +++ ; medium, ++++; strong).

\section{Results \\ 3.1. Histochemical results}

There were no histological differences between the control and corn oil groups. Therefore, the control group was taken as the basis and evaluations were made accordingly. On histopathological examination, control rat testis showed normal morphology and spermatogenesis. There normal progression of spermatogenesis with primary and secondary spermatocytes. The Sertoli cells in seminiferous tubules and Leydig cells located in interstitial connective tissue were in normal structure and numbered (Figure 1A-D).

In contrast to controls, DIBP treatment disrupted and decreased the spermatogenic cell population and caused complete spermatogenic arrest (Fig. A-I). Apoptotic and necrotic germinal cells were observed in all experimental groups (Figure 2A-I). With prolonged exposure, it was seen seminiferous tubules lined only by Sertoli cells (Figure 2G,H,I). There was an increase in vacuolization and number in Sertoli cells (Figure $2 \mathrm{H}$ ). Multinucleate giant cells were also observed within seminiferous tubules especially at high doses (Figure $2 \mathrm{~F})$. Furthermore, in DIBP treatment groups, attracted attention thickening and dilatation in basal lamina of seminiferous tubules (Figure 2C,F,I). Also, oedema and shrinkage in interstitial connective tissue (Figure 2E,G,I).

\subsection{Immunohistochemical results}

Laminin was seen in the BM of testicular tubules as a strongly stained in control group. Also laminin was detected as intense staining in spermatogonia, spermatocytes, sperm and interstitial tissue in testis sections of control group. In experiment groups 1 and 2, density of laminin staining was observed close to control group in lumen of seminiferous tubule, spermatogonia, spermatocytes, sperm and interstitial tissue. But laminin in interstitial tissue of group 3 were slightly detected than in other groups (Figure 3). If we evaluate table 1 according to staining intensity, the maximum decrease in sperm cells and the minimum decrease in Sertoli cells and Adc-L was observed.

Fibronectin was observed in the lamina propria of all groups (Fig. 4). The testicular interstitium of control group showed dense immunostaining for fibronectin while it was determined that fibronectin staining decreased in these regions in experiment groups. This decrease in fibronectin staining was more at higher doses (Figure 4). If we evaluate table 2 according to staining intensity, the maximum decrease in basal lamina of group 2 and 3 and minimum decrease in Adc$\mathrm{L}$ was seen. 


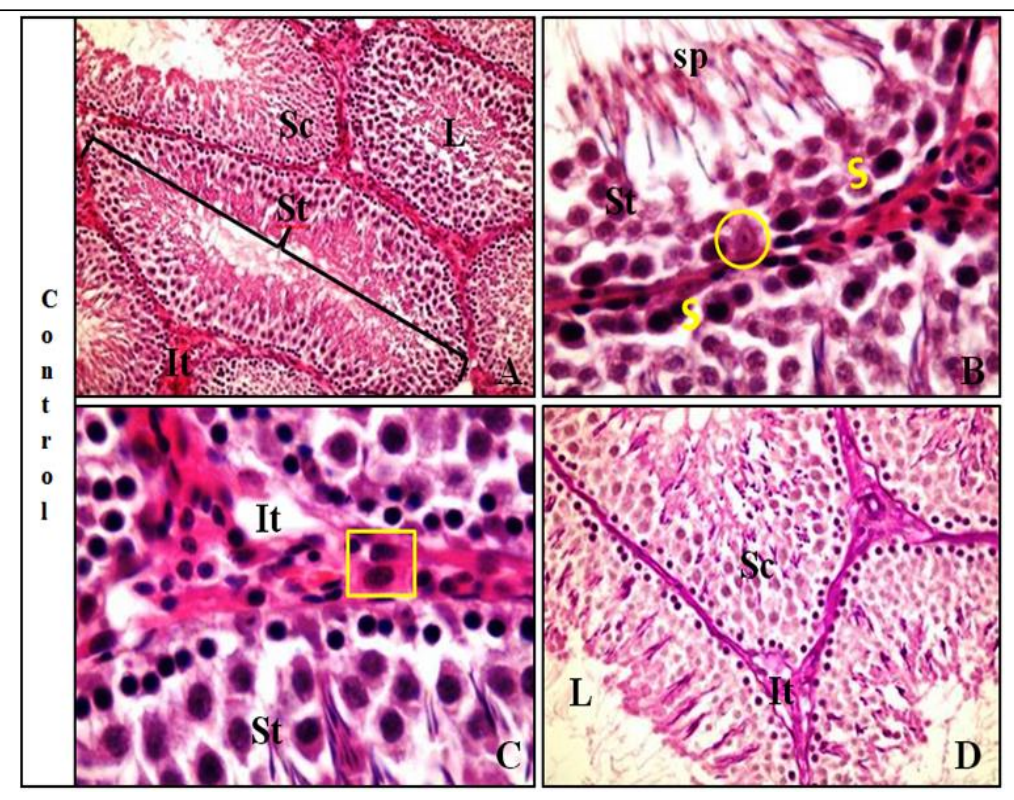

Figure 1. Testis section of control group. St; seminiferous tubules, It; interstitial tissue, S; spermatogonium, St; spermatocyte, sp; sperm, $\bigcirc$; Sertoli cell in seminiferous tubules, $\square$; Leydig cells in the interstitial connective tissue. Staining; A,B,C; H\&E, D; PAS, magnification; A;10X, B,C; 40X, D; 20X.

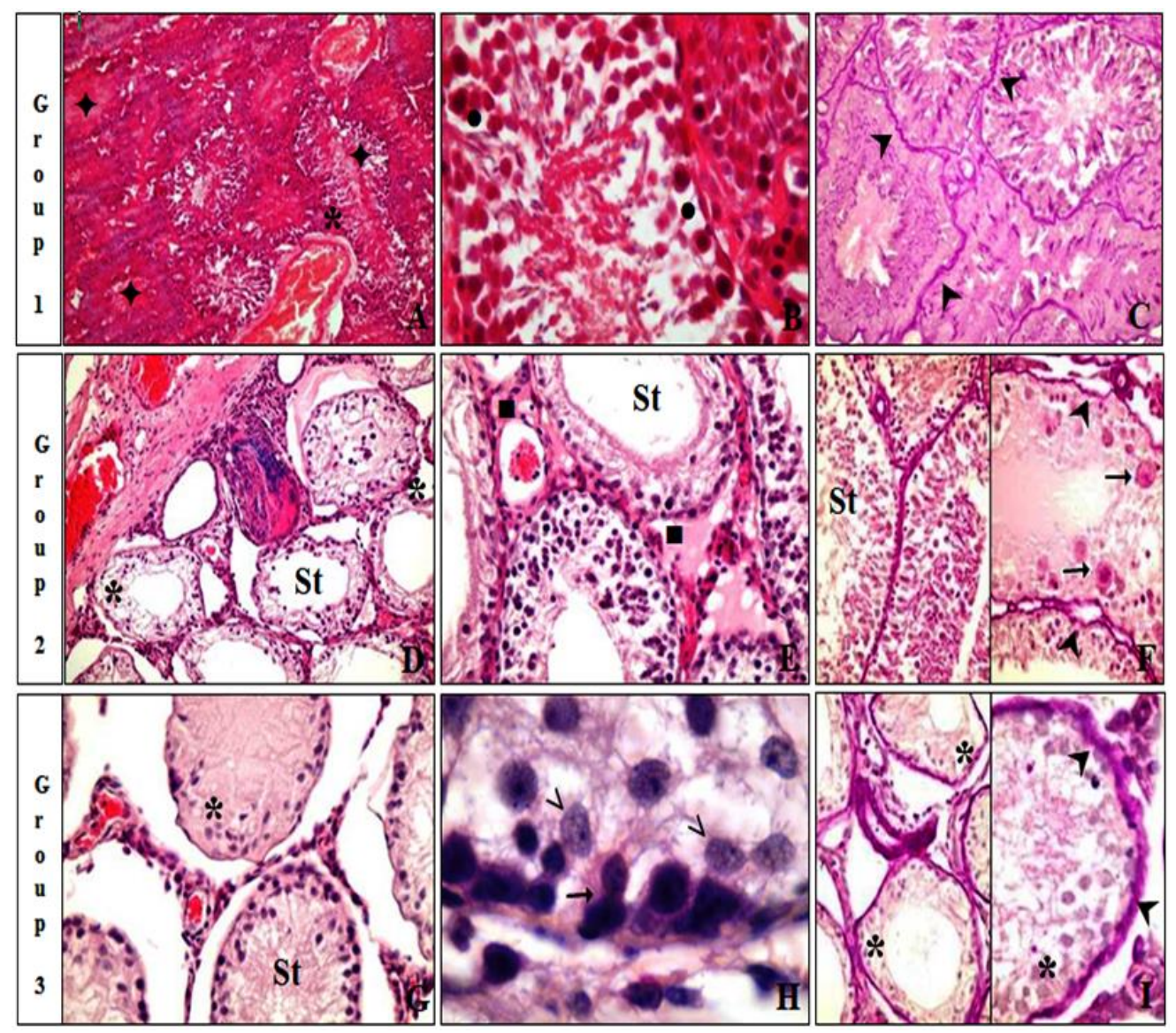

Figure 2. Testis section of experimental group. $\uparrow$; altered staining in the seminiferous tubules, *; disruption and loss/decrease in spermatogenic cell line in seminiferous tubules, $\mathbf{>}$; thickening and dilatation in basal lamina of seminiferous tubules, - ; oedema in interstitial connective tissue, st; shrinkage of the tubules, >; vacuolization in Sertoli cells, $\rightarrow$; multinucleated giant cells, $\mathbf{x}$; interstitial connective tissue. Staining; A,B,D,E,G,H; H\&E, C,F,I; PAS, magnification; A;10X, B; 40X, C,E,G; 20X, H;100X, F; 40X, I; left figure 20X-right figure 40X. 
Table 1. Immunohistochemical reactions to laminin expression in testis. - ; no reaction, +; very weak reaction, ++; weak reaction, +++; medium reaction, ++++; strong reaction. 0; no spermatocyte, spermatid, sperm, Spmt: Spermatogonium, Spct; spermatocyte, Spmd; spermatid, Adc-L; adluminal compartment-lumen, It; interstitial tissue.

\begin{tabular}{|l|c|c|c|c|c|c|c|c|}
\cline { 2 - 9 } \multicolumn{1}{c|}{} & \multicolumn{7}{c|}{ Laminin } \\
\cline { 2 - 10 } & Spmt & Spct & Spmd & Sperm & Sertoli & $\begin{array}{c}\text { Basal } \\
\text { lamina }\end{array}$ & Adc-L & It \\
\hline Control & ++++ & +++ & ++++ & ++++ & ++++ & ++++ & ++++ & ++++ \\
\hline Group 1 & +++ & +++ & +++ & ++ & +++ & +++ & +++ & ++ \\
\hline Group 2 & +++ & ++ & 0 & 0 & +++ & ++ & +++ & ++ \\
\hline Group 3 & ++ & 0 & 0 & 0 & +++ & ++ & +++ & + \\
\hline
\end{tabular}

Table 2. Immunohistochemical reactions to fibronectin expression in testis. - ; no reaction, +; very weak reaction, ++; weak reaction, +++; medium reaction, ++++; strong reaction. 0; no spermatocyte, spermatid, sperm, Spmt: Spermatogonium, Spct; spermatocyte, Spmd; spermatid, Adc-L; adluminal compartment-lumen, It; interstitial tissue.

\begin{tabular}{|l|c|c|c|c|c|c|c|c|}
\cline { 2 - 10 } \multicolumn{1}{c|}{} & \multicolumn{7}{c|}{ Fibronectin } \\
\cline { 2 - 10 } & Spmt & Spct & Spmd & Sperm & Sertoli & Basal & Adc-L & It \\
& lamina & & \\
\hline Control & ++++ & +++ & ++++ & ++++ & ++++ & +++ & ++++ & +++ \\
\hline Group 1 & ++ & + & + & + & ++ & + & ++ & +++ \\
\hline Group 2 & + & + & 0 & 0 & ++ & - & ++ & + \\
\hline Group 3 & + & 0 & 0 & 0 & + & - & ++ & + \\
\hline
\end{tabular}



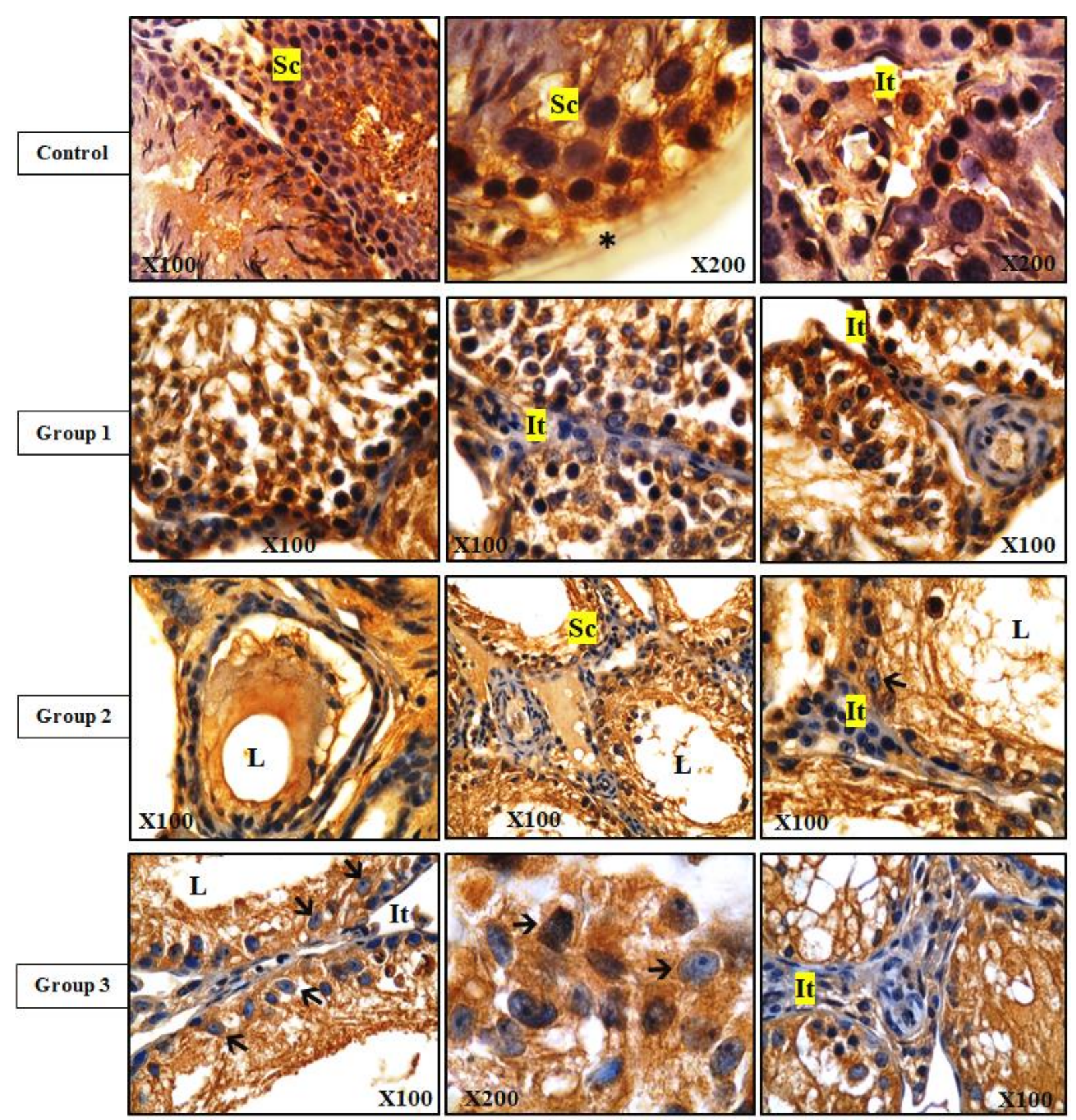

Figure 3. Immunohistochemical staining for laminin in control and experimental testes tissue. Laminin is founded mostly in adluminal compartment, spermatogenic cells and innermost part of the lamina propria of seminiferous tubules. L: lumen, It: interstitial tissue, Sc: Spermatogenic cells, arrow: sertoli cell, $\boldsymbol{*}$ : basal membrane. 

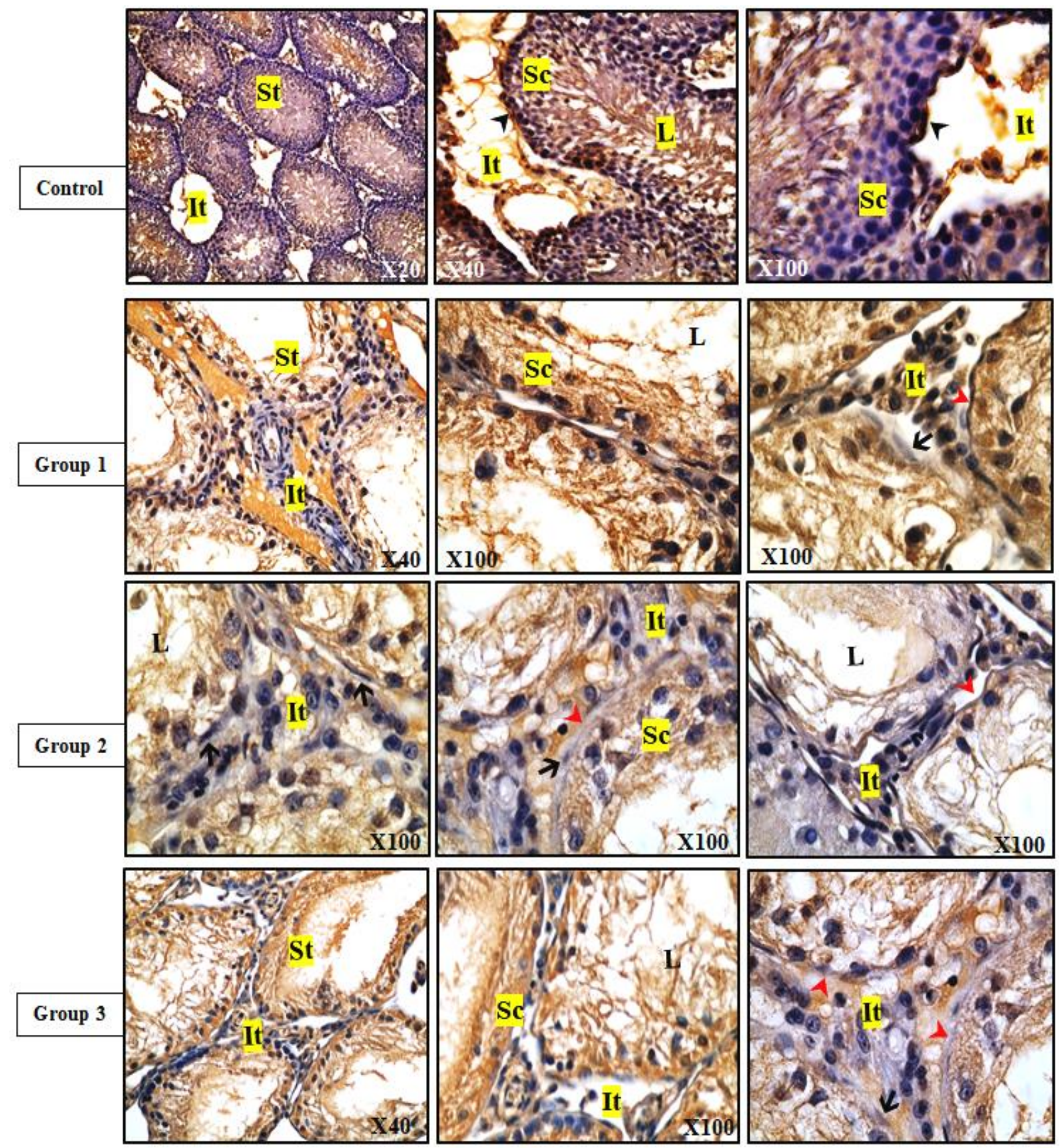

Figure 4. Immunohistochemical staining for fibronectin in control and experimental testes tissue. Fibronectin is founded mostly in interstitial tissue of control group. In experimental group (especially group III), fibronectin is sligthly decreased. L: lumen, It: interstitial tissue, Sc: Spermatogenic cells, arrow: degenerative myoid cell, arrow head: basal membrane.

\section{Discussion}

It known that spermatogenesis is a specific process of proliferation and differentiation of germ cells in testes. Cell-cell interactions occurring between germ cells at different stages of differentiation within the seminiferous tubule are very important. Intercellular junctions consist of specific proteins involved in the adhesion, attachment, and communication between adjacent cells [11]. Therefore, these proteins play an important role in the coexistence of cells.
Toxicants acting cell generally result in a similar pattern of morphological changes. In this study, in testes tissue of rats exposed to DIBP were observed similar morphological results. It was observed that DIBP treatment disrupted the spermatogenic cell population. This is due to the fact that DIBP directly affects cell division and reduces division in spermatogenic cells.

Different germ cell populations each display their own sensitivity to toxicants. Characteristic of germ cellspecific toxicity is rapid apoptosis and phagocytosis of the affected cells by the Sertoli cell [12]. As a matter of 
fact, apoptotic and necrotic germinal cells were observed in all experimental groups of this study. It can be said that this increase in the Sertoli cells, which are observed in a large number in the experimental groups, is for phagocytosis of dead cells.

Vacuolization is one of the most common morphological responses of the Sertoli cell to injury. Most of the vacuoles appear to be caused by the dilated cisternae of endoplasmic reticulum when ultrastructural examination [12]. Vacuolization in Sertoli cells cytoplasm after exposed DIBP. This situation demonstrates that DIBP damage the organelle structure of Sertoli cells.

It is reported that exposure to some phthalate esters causing germ cell loss and increasing the number of multinucleated germ cells [13]. Multinucleate giant cells of germ cell origin were also observed within seminiferous tubules especially at high doses in this study. Multinucleated giant cell formation may be related germinal cell degeneration. It was found degenerative features such as multinucleated giant cell formation, germ cell vacuolization or cell-specific degeneration and depletion of germ cells depending upon the DIBP, in short-duration this study (28 days).

Interstitial fluid and seminiferous tubule fluid, secreted by the testis. This fluid can be readily disturbed, resulting in a variety of effects, including tubular lumenal dilatation, tubular lumenal contraction, and interstitial oedema [14]. In DIBP treatment groups of this study were seen attention thickening and dilatation in basal lamina of seminiferous tubules, oedema and shrinkage in interstitial connective tissue. Like some other chemicals, DIBP may have disturbed blood flow and fluid balance by causing testicular structure change.

It has been shown that ECM has a role in germ cell functions in the Sertoli and Seminiferous epithelium and in the blood-testis barrier dynamics [15]. In this study, basal lamina thickening after DIBP administration may be a structural change to strengthen the blood-testis barrier in order to reduce the passage of DIBP from blood.

Fibronectin are secreted by peritubular cells while laminin were secreted by Sertoli cells [16]. The laminin play an important role in adhesion of gonocytes to the lamina densa and adjacent Sertoli cells before their transition from basal compartment to adluminal compartment [17].

Laminin biosynthesis by the spermatogonia is regulate the cell adhesivity to the basement membrane of the seminiferous tubules. Changes affecting the relation between Sertoli cells and spermatogonia and, in particular, decreased adhesivity to the basement membrane and may be cause spermatogonia to move from the basal to the adluminal compartment [17].

In this study, a large number of spermatogenic cells were observed in the lumen of the seminiferous tubule after DIBP exposure. Besides an increase in Sertoli cells was observed in tubules especially in groups 2 and 3. The cause of the increase in the number of Sertoli cells observed in the experimental groups may be the prevention of loss of spermatogenic cells. In short, we can also say that the number of Sertoli cells is increased for more laminin production. As a matter of fact, there has not been much change in laminin density in luminal compartment of seminiferous tubules.

Fibronectin involvement was clearly observed in the basal membrane of the seminiferous tubule and germinal cells (spermatocytes, spermatids and especially in head parts of spermatozoa, basement membrane, myoid cell layer, and interstitial area). The intense observation of fibronectin in these regions suggests that it may be effective in cell-cell and cellmatrix interaction in spermatogenesis $[18,19]$.

The deficiency in fibronectin, like histological lesions of testes exposed to different other phthalates, might be due to either extrinsic or intrinsic causes. Myoid cells are known to synthesize fibronectin [20]. After DIBP administration, damage seen in some myoid cells, especially in the high dose group, may be one of the causes of fibronectin decrease in the interstitial tissue. In this study, fibronectin involvement, especially in the basal membrane of seminiferous tubules and in the interstitial tissue decreased after DIBP administration. When consider the role of testicular fibronectin in contributing to connective tissue and seminiferous tubule structure, cell-basal membrane and basement membrane-interstitial tissue interaction, it can be said that it does not perform all these functions after exposure to DIBP.

Based on the data obtained from this research, it can be said that DIBP causes irreparable damage to rat testicular tissue, especially in terms of spermatogenesis. Different studies need to be done on this subject, but according to the results of this research, it can be said that DIBP can cause infertility in men. For this reason, I recommend the use of other alternative plasticizers whose safety is determined.

\section{Acknowledgments}

I thank the Adnan Menderes University for support of this research through grant number FEF-2014/018. Also, I thank Merve Aky1ldı, master student, for the contribution of the laboratory work.

\section{Ethics}

There are no ethical issues after the publication of this manuscript.

\section{References}

1. Chemical Book, Diisobutyl phthalate Chemical Properties, Uses, Production.

https://www.chemicalbook.com/chemicalproductproperty 2017 (accessed 15.06.2017). 
2. Environmental protective Agency (EPA). PhtalatesDiisobutylphthalate, Biomonitoring, America's Children and the Environment, United States, Third Edition, 2017, pp 168-356.

3. Barlow, NJ, Foster, PM. 2003. Pathogenesis of male reproductive tract lesions from gestation through adulthood follwing in utero exposure to di(n-butyl) phytalate. Toxicolology; 31, 397-410.

4. Veeramachaneni, DN, Parks, L. 2000. Perinatal exposure to the phytalates DEHP, BBP and DINP but DEP, DMP or DOTP, alters sexual diffrentiation of the mal erat. Toxicological Sciences; 58 , $350-365$

5. Borch, J, Ladefoged, O, Vinggaard, AM. 2004. Steroidogenesis in fetal male rats is reduced by DEHP and DINP, but endocrine effects of DEHP are not modulate by DEHA in fetal, prebubertal and adult male rats. Reproductive Toxicology; 18, 53-61.

6. Parks, LG, Ostby, JS, Lambright, CR, Abbot, BD, Klinefelter, GR, Barlow, NJ, Gray, JLE. 2000. The plasticizer diethylhexyl phytalate induces malmormations by decreasing fetal testosterone synthesis during sexual differentiation in the male rat. Toxicological Sciences, 58, 339-349.

7. Bosman, FT, Stamenkovic, I.2003. Functional structure and composition of the extracellular matrix. Journal Pathology, 200, 423-428.

8. Humphries, MJ. 2000. Integrin structure, Biochemical Society Transactions; 28, 311-339.

9. OECD, Guidelines for Testing of Chemicals No: 407. Repeated Dose 28-day Oral Toxicity Study in Rodents, Organization for Economic Cooperation and Development, 1995, Paris, France.

10. Bancroft, JD, Cook, HC. Manual of Histological Tecniques and Their Diagnostic Application. New York, Churchill Livingstone, 1994, p 457.
11. Cheng, CY, Mruk, DD. 2002. Cell junction dynamics in the testis: Sertoli-germ cell interactions and male contraceptive development. Physiological Reviews; 82, 825-74.

12. Creasy, DM. 2001. Pathogenesis of male reproductive toxicity. Toxicologic Pathology; 29(1), 64-76.

13. Spade, D.J, Hall, S.J, Wilson, S, Boekelheide, K. 2005. Di- $n-$ Butyl Phthalate Induces Multinucleated Germ Cells in the Rat Fetal Testis Through a Nonproliferative Mechanism. Biology of Reproduction; 93(5), 110.

14. Setchell, BP. 1990. Local control of testicular fluids. Reproduction Fertility and Development; 2, 291-309.

15. Cheng, CY, Siu, MKY. 2008. Extracellular matrix and its role in spermatogenesis. Advances in Experimental Medicine and Biology; 636, 74-91.

16. Skinner, MK. 1991. Cell-Cell Interactions in the Testis. Endocrine Reviews; 12, 45-77.

17. El Quali, H, Leheup, BP, Gelly, JL, Grignon, G. 1991. Laminin ultrastructural immunolocalization in rat testis during ontogenesis. Histochemistry; 95, 241-246.

18. Shaller, J, Glander, HJ, Dethloff, J. 1993. Evidence of $\beta 1$ integrins and fibronectin on spermatogenic cells in human testis. Human Repropduction; 8, 1873-1878.

19. Nacar, E, Elmas, Ç, Erdoğan D. 2014. Localization of $\beta 1$ integrin and fibronectin on spermatogenic cells in rat testis. Mustafa Kemal Üniversitesi Tip Dergisi; 5(20), 28-37.

20. Santamaria, L, Martinez-Onsurbe, $\mathrm{P}$, Paniagua, R, Nistal, M 1990. Laminin, type IV collagen and fibronectin in normal and cryptorchid human testes. An immunohistochemical study. International Journal of Andrology; 13, 135-14. 